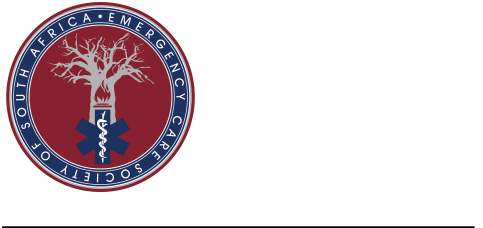

ARTICLE INFORMATION

Received: 17 July 2020

Revision received: 28 August 2020

Accepted: 21 November 2020

Keywords:

FOAMed

Continuous Professional Development online learning

asynchronous learning

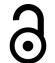

This open access article is distributed under Creative Commons licence CCBY-NC 4.0

ISSN:2709-3786

\section{Using Free Open Access Medical Education (FOAMed) for Emergency Medical Service education}

Judy Sheahan ${ }^{1, *}$, Susan van Schalkwyk ${ }^{1}$, Lianne Keiller ${ }^{2}$, Enrico Dippenaar ${ }^{3,4}$

${ }^{1}$ Centre for Health Professions Education, Faculty of Medicine and Health Sciences, Stellenbosch University

${ }^{2}$ Academic ICT, Information and Communication Technology, Stellenbosch University

${ }^{3}$ Division of Emergency Medicine, Faculty of Health Sciences, University of Cape Town

${ }^{4}$ Emergency Medicine Research Group, Anglia Ruskin University

*Corresponding author: judysteyn@hotmail.com, P O Box 241, Cape Town, 8000

\section{ABSTRACT}

Background: Continuous professional development (CPD) is a registration requirement for all healthcare professionals in South Africa. The shift-driven work environment of emergency and critical care providers often means it is logistically impractical to attend CPD events. FOAMed and online learning could make CPD activities more accessible. The aim of the study was to determine to what extent available FOAMed resources could be used to supplement the outcomes for successful completion of a CPD course.

Methods: A retrospective content analysis was used to review FOAMed resources posted within a two-month period for their ability to cover outcomes of the Ambulance Emergency Assistant (AEA) refresher course (a CPD course). Data were subject to a three-step process of content analysis that was matched to 17 identified course outcomes (themes).

Results: A total of 441 FOAMed posts were reviewed during a twomonth period. Of the 441 posts, 269 (61\%) were excluded, and 172 (39\%) FOAMed posts were determined relevant to cover the 17 identified themes of the AEA refresher course. The two most frequently observed themes were related to (i) professionalism, with 45 (26.2\%) posts, and (ii) other general medical emergencies, with 39 (22.7\%) posts. The remaining 15 themes had $88(51.1 \%)$ posts distributed between them.

Conclusion: The study highlights the potential of FOAMed resources to supplement the delivery of education, such as a CPD course. Given that the outcomes were unequally represented, educational institutions should be aware that despite the value of FOAMed, due caution must be given to the critiques and limitations of this educational approach.

Sheahan J, van Schalkwyk S, Keiller L, Dippenaar E. Using Free Open Access Medical Education (FOAMed) for Emergency Medical Service education. South African Journal of Pre-hospital Emergency Care. 2020; 1(2):13-21. doi:10.24213/1-2-4196 


\section{BACKGROUND}

Free open access medical education (FOAMed) is a term that has become well established in emergency medicine and critical care communities. ${ }^{1-4}$ The term is recognised as a collection of freely available educational material and information, which is ideal as a tool for asynchronous learning. Since its inception in 2012, many websites have become known as reliable contributors to FOAMed. ${ }^{4}$ FOAMed has also been evolving as a model to support continuous professional development (CPD). ${ }^{1,2}$ FOAMed resources are easily accessible and portable, which make them ideal for healthcare professionals wishing to strengthen their knowledge and skills when it best suits their schedules. FOAMed resources are distributed via various social media platforms such as podcasts (audio), vodcasts (video) and blogs (written), and has the potential to shorten the knowledgetransition gap between inception, publication and bedside application. ${ }^{2,5}$ The prediction is that new and updated medical modalities could be implemented at the patient's bedside sooner, and by embracing internet technologies and social media learning, healthcare professionals could enhance the dissemination of peer-reviewed medical knowledge faster, and with potentially improved accuracy. ${ }^{3,4,6}$ These benefits of FOAMed have undoubtedly been put to use during the ongoing COVID-19 pandemic. $^{7}$

Worldwide, clinicians are being forced to take care of critically ill patients without the luxury of applying tried and tested controlled trials. Instead, clinicians have been able to share experiences, review available literature and share updated clinical practice guidelines in a timely manner with colleagues around the world via online platforms like FOAMed. ${ }^{7}$ The converse of this positive effect of accessible and timely discussions between colleagues, is the impact that online accounts with large followings could have when they criticise or discriminate publicly against FOAMed content with which they disagree. Nonetheless, the authors believe that discussion elicited among professionals can strengthen the quality of these FOAMed resources.

Internet technologies and social media have become powerful tools for the distribution of medical knowledge, allowing its users to access information when, where and how they want.1,8 This has created opportunities and new ways in which learning can be facilitated, and could even provide alternative means to facilitate CPD activities. Social media is also emerging as a field of scholarship in medical education, offering many opportunities for innovation. ${ }^{8}$ It is reported that learner engagement, collaboration and professional development all transpire through opportunities provided by learning via social media. ${ }^{8}$ Studies evaluating learning via social media mostly return positive findings, reporting individualised learning opportunities, increased learner satisfaction, widespread applicability, accessibility, timeous and cost-effective facilitation of learning. 9,10 At the time of this study, only two other studies had specifically evaluated undergraduate curriculum content covered with FOAMed resources, and they yielded similar results, highly recommending that FOAMed offers much to facilitate learning. Yet it should not be used as the only resource in undergraduate studies, but rather used to supplement existing knowledge and traditional learning. ${ }^{11,12}$ Subsequently, as FOAMed has evolved, more of these curriculum evaluations have been conducted, and the results remain consistent. $^{13}$

The healthcare profession is dynamic in that it is ever-evolving, changing and responding to shifting healthcare imperatives, as evidenced by the ongoing global pandemic. ${ }^{1,2,14}$ Technological advances, globalisation and changes in the world's health needs represent only a few reasons why healthcare professionals need to be lifelong learners and continue their professional development. ${ }^{3}$ Working in an emergency and critical care setting requires providers to remain current and competent in life-saving interventions like resuscitation and trauma care. This notion is supported by resuscitation and trauma organisations worldwide, where new and updated guidelines are released every five years. ${ }^{1}$ These guidelines provide practising emergency care providers with the latest recommended evidence-based medicine and best practice treatment strategies.

Working in a shift-driven environment often poses a challenge to emergency and critical care providers to find the time to stay up to date with the latest recommended medical care or attend CPD activities. CPD refers to any activities that support lifelong learning, enhancing the competence (knowledge, attitudes and skills) of medical practitioners. ${ }^{15,16}$ One way in which CPD is addressed in the medical profession is through conferences; however, work from the United States of America (USA) reported that on average only $25 \%$ of medical residents were able to attend conferences, with noticeably late arrivals and requests to leave early. ${ }^{14,17}$ Although not specifically referring to emergency care providers, medical residents comparably work in a shift-driven environment. This may be an indication of the need for more flexible or alternative means of delivering CPD events in the medical profession. Dur- 
ing 2020, many healthcare conferences and medical gatherings were cancelled or postponed due to COVID-19, and with these sudden global changes, FOAMed, with its flexible and accessible modes of delivery, could prove invaluable in the dissemination of life-saving medical knowledge.

In South Africa, CPD is a registration requirement instituted by the national health professions regulator, the Health Professions Council of South Africa (HPCSA). This is applicable to a majority of healthcare professionals in South Africa, including Ambulance Emergency Assistants (AEA), who fall into the middle (intermediate) level of the emergency care provider hierarchy in South Africa. The pre-hospital service in South Africa broadly consists of three levels - basic, intermediate and advanced. The basic and intermediate level certifications are obtained from a short-course study at accredited training colleges, while in the advanced level there are a variety of formal qualifications, including a certificate, national diploma and bachelor's degree. Basic level practitioners are supervised practitioners, but from the intermediate level, practitioners are considered autonomous and independent. Alongside autonomous practice at the intermediate level is the expanded scope of practice, which includes some invasive procedures like intravenous cannulation and the administration of a variety of emergency medications. All practitioners are strongly encouraged by the HPCSA to remain current with CPD compliance as quality assurance is maintained through random audits. Deregistration from the HPCSA or fines may apply if non-compliance is found to be present. $^{18}$

The AEA refresher programme is an example of an existing, accredited CPD activity comprising 17 course outcomes and capabilities as directed by the HPCSA. ${ }^{18}$ The refresher course is the most common CPD activity available to AEA practitioners in South Africa. This accredited five-day classroombased course aims to provide the practitioner with a revision of knowledge gained during their original training, an opportunity to practice skills not commonly used in everyday practice, and inform the practitioners of any treatment guideline updates or changes. AEA practitioners are expected to achieve cognitive, educational objectives, appropriate to the first three levels of Bloom's Taxonomy; these are knowledge (remember), comprehension (understand), and application. ${ }^{19}$ Practitioners should therefore not only know and understand the fundamentals of emergency care, but have the ability to apply this knowledge in their practice.

Anecdotal evidence suggests that the AEA re- fresher course is not well attended due to constraints like having to take time off work, and having to travel and find accommodation near educational institutions. It may potentially be to patients' detriment if these practitioners are not up to date with the latest treatment recommendations and guidelines. It can be argued that in this context, FOAMed resources could contribute to the knowledge of these practitioners. As previously mentioned, these resources are easily accessible, portable and have the potential to accelerate bedside application.

To effectively use FOAMed resources as a potential supplement to traditional educational delivery, it must first be known to what extent FOAMed resources are able to cover the required course outcomes. The authors chose the AEA refresher course as the subject for review due to the scope of practice including more invasive patient procedures and independently practising clinicians, when compared to the basic pre-hospital practitioner in South Africa. The advanced pre-hospital clinician scope of practice was excluded due to the time constraints associated with this study at the time.

\section{DESIGN AND METHODS}

This study utilised a retrospective content analysis methodology to review FOAMed resources posted within a two-month period for their ability to cover the outcomes of the AEA refresher course. ${ }^{20}$ For the purpose of this study, a content analysis was appropriate as it allowed the authors to restructure the qualitative data (themes) into quantitative data (frequency of the themes). ${ }^{21}$

The data for this study were obtained from the FOAM EM ${ }^{22}$ website, as a curator site for all \#FOAMed posts. This means that all posts (including published articles, blogs, podcasts and videos) with the '\#FOAMed' characteristic link directly to the site in real-time. This was beneficial as the primary researcher did not have to search for new daily content manually, and it was a suitable way to ensure that no correctly tagged FOAMed posts were overlooked. At the time of data collection, the feed from FOAM EM was collating posts from over 250 FOAM sites, which represented about $70 \%$ of all known contributing FOAMed websites; according to Cadogan, in March 2016, 356 websites were listed as contributors. ${ }^{23}$ All resources posted to FOAM EM from 1 March to 30 April 2017 were reviewed. The FOAM EM curator site no longer exists, but a similar function is now offered by FOAMed contributor Life in the Fast Lane. ${ }^{24}$

All resources posted during the two-month study 
period in English, without restriction and for free, were included in the study. Resources were excluded if the content extended beyond the capabilities of the AEA practitioner (as described by the 17 AEA refresher course outcomes); duplicate posts were also excluded. Ethical approval was not required for this study as no primary participants were involved, and all the data were publicly available.
A subscription email account was used to access the FOAMed posts and receive a daily email with links to newly published FOAMed posts. In keeping with the selected approach of content analysis, each of the collected FOAMed posts was reviewed to determine relevance to cover the required 17 outcomes of the AEA refresher course. Figure 1 represents the initial sorting that each of the resources underwent after being received via email, up to inclusion for analysis.

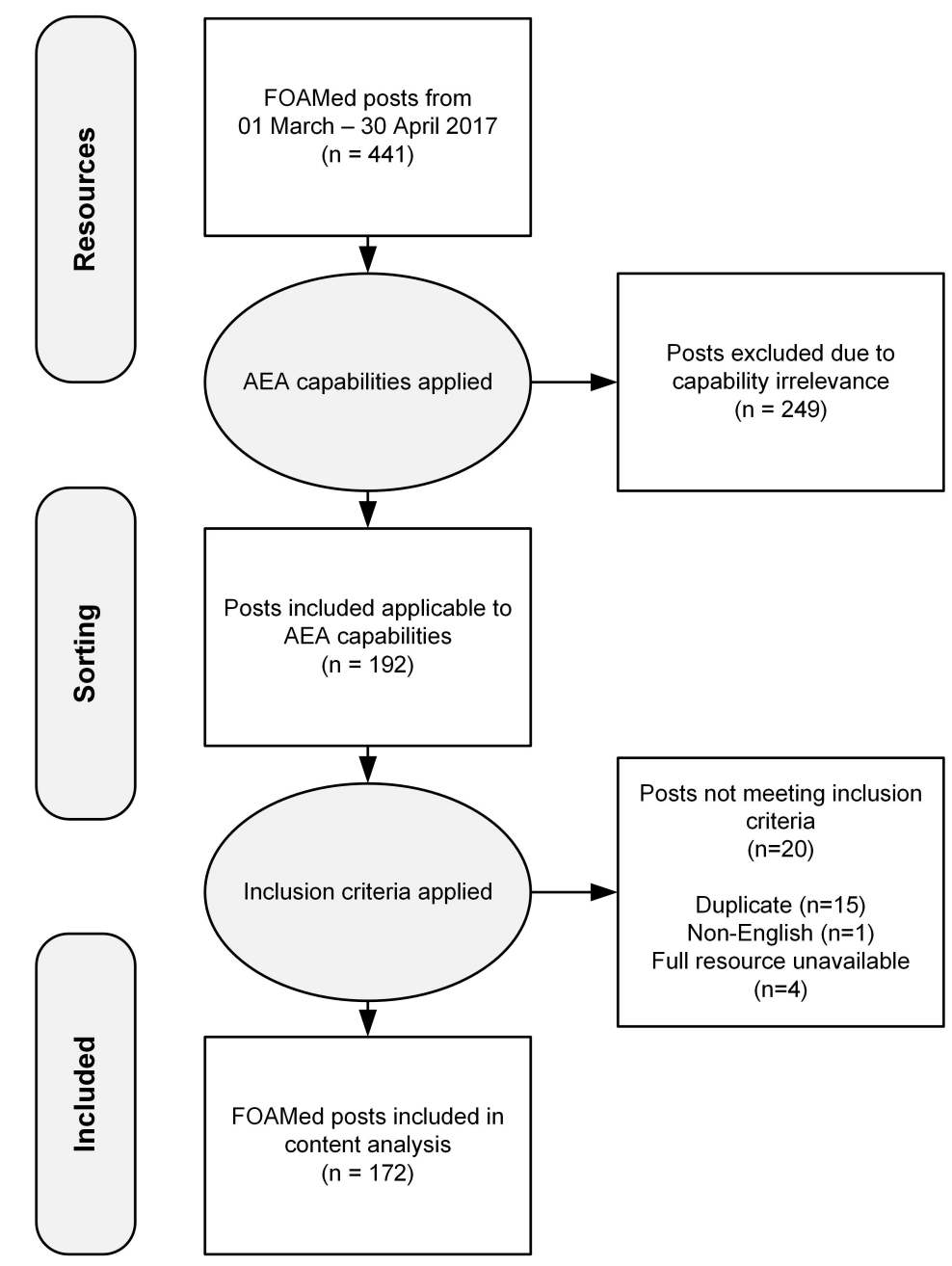

Figure 1: Review of all FOAMed posts published from 1 March to 30 April 2017

All 441 collected FOAMed posts underwent a three-step process of analysis: (i) the resource title was viewed and included or excluded based on the AEA capabilities and inclusion criteria. (ii) Included resources were fully read/listened to/viewed (depending on the type of resource, i.e. blog, podcast, video, etc.) and matched to coded themes 1-17. (iii) Lastly, resources were categorised by medical competency and Bloom's Taxonomy cognitive domain. ${ }^{19}$ In addition, the media type which each resource represented was recorded. This information could be useful when alternative educational strategies (i.e. blended or online learning activities) are prepared. If the type of media is known, wider audiences could be addressed by appropriately including various types of media to suit different types of learners (i.e. auditory or visual). This, in turn, can positively contribute to learning via social media and FOAMed, since resources would be available in various forms and when it best fits into the practitioners' everyday life. 


\section{Data Analysis}

All data resources were collected, sorted, analysed and recorded by the primary author (single researcher) into a Microsoft Excel® spreadsheet. Basic statistic functions in Microsoft Excel ${ }^{\circledR}$ were utilised to convert the themed information into quantitative data (percentages). A sample of the data was randomly extracted for review by an independent colleague for accuracy. Less than $1 \%$ error was found, and all the data were included in the final review.

Table 1: AEA refresher course outcomes covered by FOAMed posts $(\mathrm{N}=172)$

\begin{tabular}{|c|c|}
\hline Theme & Total Posts \\
\hline Ethics in the EMS & 8 \\
\hline Child and elder abuse & 0 \\
\hline $\begin{array}{l}\text { Professionalism (characteristics of a professional, documentation, professional } \\
\text { well-being) }\end{array}$ & 45 \\
\hline Law (patient rights, regulatory bodies) & 2 \\
\hline Haemorrhagic-, anaphylactic- and neurogenic shock & 5 \\
\hline $\begin{array}{l}\text { Concussion, closed head injuries (RICP), base of skull fracture and neuroprotective } \\
\text { strategies }\end{array}$ & 4 \\
\hline $\begin{array}{l}\text { Open pneumothorax, tension- and pneumothorax, haemothorax, flail chest and } \\
\text { cardiac tamponade }\end{array}$ & 3 \\
\hline Spinal Injuries, spinal motion restriction & 4 \\
\hline Limb \& pelvic fractures, amputations, rhabdomyolysis, the use of tourniquets & 8 \\
\hline Burns (types and severity) & 2 \\
\hline Asthma, chronic obstructive pulmonary disease (COPD) and pneumonia & 13 \\
\hline $\begin{array}{l}\text { ACS (myocardial infarction and angina), chest pain, heart failure and 3-lead ECG } \\
\text { review }\end{array}$ & 11 \\
\hline Seizures and cerebrovascular attack (CVA) & 9 \\
\hline $\begin{array}{l}\text { Hypoglycaemic emergencies, DKA, abdominal emergencies, other medical emer- } \\
\text { gencies }\end{array}$ & 39 \\
\hline Epiglottitis, croup and dehydration in paediatrics & 9 \\
\hline $\begin{array}{l}\text { Resuscitation (shockable and non-shockable arrest), PCA management and decla- } \\
\text { ration of death }\end{array}$ & 9 \\
\hline $\begin{array}{l}\text { Placenta Previa, placenta abruptio, } \mathrm{PPH} \text {, pre-eclampsia, eclampsia, prolapsed cord } \\
\text { presentation, breech presentation, new-born care }\end{array}$ & 1 \\
\hline
\end{tabular}

AEA = Ambulance Emergency Assistant; FOAMed = Free Open Access Medical Education; EMS = Emergency Medical Services; RICP = Raised intracranial pressure; ACS = Acute coronary syndromes; ECG = Electrocardiogram; DKA = Diabetic Ketoacidosis; PCA $=$ Post-cardiac arrest; PPH $=$ Post-partum haemorrhage

\section{RESULTS}

A total of 441 FOAMed posts were reviewed during the study period. Of the 441 posts, $269(61 \%)$ were excluded due to their focus extending beyond the AEA capabilities. The remaining 172 (39\%) FOAMed posts were deemed relevant to the 17 identified outcomes of the AEA refresher course. Table 1 demonstrates an aggregated representation of the 17 course outcomes and the corresponding FOAMed coverage of these outcomes.

The two most frequently observed themes were related to professionalism, with 45 posts, and other general medical emergencies, with 39 of 172 posts. There were seven themes (ethics, limb injuries, respiratory, cardiovascular and neurological emergencies, paediatrics and resuscitation) with average $(5-8 \%)$ representation, and the remaining eight themes each had very low $(<3 \%)$ representation. Professionalism encompassed characteristics and attitudes of practitioners and professional wellbeing. ${ }^{18}$

Additional analysis of the FOAMed posts revealed that the majority $(67.44 \%)$ of posts were in the form of a blog, $79.07 \%$ fell into the knowledge discipline of a medical professional's competency, and $62.79 \%$ of posts were deemed applicable to the third tier (application) of Bloom's Taxonomy. Table 2 gives a complete representation of the additional analysis per element.

\section{DISCUSSION}

The aim of this study was to determine the extent to which available FOAMed resources could 
be used to cover the outcomes of a CPD course in the hope that the results could inform alternative teaching and learning methods in the delivery of the course.

Table 2: Representative Data Analysis per Element

\begin{tabular}{lll}
\hline Type of Media & Number & $\mathbf{\%}$ \\
\hline Blog & 116 & 67.44 \\
Podcast & 35 & 20.35 \\
Article & 16 & 9.30 \\
Video & 3 & 1.74 \\
Vodcast & 2 & 1.16 \\
\hline Competency & Number & \% \\
\hline Knowledge & 136 & 79.07 \\
Attitude & 31 & 18.02 \\
Skills & 5 & 2.91 \\
\hline Bloom's Taxonomy & Number & $\mathbf{\%}$ \\
\hline Application & 108 & 62.79 \\
Knowledge & 61 & 35.47 \\
Comprehension & 3 & 1.74 \\
\hline
\end{tabular}

The potential influence of FOAMed is considerable, as identified by this study, and even more so recently with the dissemination of rapidly evolving information related to COVID-19.7 It provides daily posts containing information relating to trending topics in emergency medicine and changing trends as new research and experiences emerge globally. As a resource, it has become popular in the emergency medical care environment in light of the quantity and quality of information it provides. ${ }^{1-4}$ Emergency and critical care specialists were able to share valuable experiences of patients with COVID-19, and these results were utilised by researchers and laboratories worldwide to recommend and continuously update the latest innovations and treatment modalities. ${ }^{7}$ It would be unwise not to embrace the positive impact that FOAMed has had in the emergency and critical care environment.

The FOAMed resources explored in this study were seen to address more than one-third of the AEA course outcomes. The course outcomes were, however, unequally represented by FOAMed resources and just over half of the resources were excluded due to their focus extending beyond the AEA practitioners' capabilities. The AEA practitioners' capabilities include mostly basic resuscitation, with only some invasive procedures. Their capabilities do not include interventions like advanced airway management and ventilation strategies, 12-lead ECG analysis, blood gas analysis, and emergency sonography, which are topics frequently trending among FOAMed posts as most of the major contributors and experts are emergency- and trauma physicians or surgeons. ${ }^{25}$ In addition, the resources are aimed at a global audience, and therefore likely to under-represent specific course curricula in the South African context. While FOAMed is potentially a valuable resource, educators and facilitators would still need to curate foundational content and monitor trending topics to determine those that could serve as educational supplements.

This study found that while FOAMed can supplement foundational knowledge, it cannot entirely replace core curricular content. Nevertheless, it could be argued that this resource could indeed add value when focusing on certain key aspects of the curriculum, for example, professionalism, the topic with the most extensive coverage by FOAMed. Clinicians often become so overly immersed in clinical information that they disregard attitudinal issues and their personal or professional well-being, although these are of equal importance. A competent medical professional should possess equal knowledge, attitude and skill. ${ }^{15,16}$ It should be reassuring to medical practitioners and employers that FOAMed not only focuses on clinical emergency medical care, but also places emphasis on the non-clinical dimension of being a healthcare professional and the individual providing the care.

This study revealed that (i) the majority of FOAMed resources reviewed were blogs; (ii) they contributed knowledge to the overall competence required from healthcare providers; and (iii) they mostly represented the application tier of Bloom's Taxonomy. ${ }^{19}$ Application of knowledge is critical to practitioners because they are required to practice independently and apply this knowledge in their clinical environment. These findings emphasise that FOAMed resources can add value to the delivery of CPD, like the AEA refresher course. Often, CPD activities focus primarily on dissemination of knowledge with skills practice and application of the knowledge alongside that. ${ }^{15,16}$ Knowledge thus remains the foundation of professional development and lifelong learning.

Healthcare providers using the information shared on FOAMed must remain aware of its limitations. FOAMed is often critiqued because it is not conventionally peer-reviewed prior to publication. However, recently, it has been debated that the reliability of FOAMed resources is increasing with the interactions they receive via social media and its non-conventional and 'open' peer-review process. ${ }^{1,25-27}$ Recently, the blog Academic Life in Emergency Medicine (ALiEM) introduced a process of 'expert and peer review' whereby feedback, commentary and edits are given on published arti- 
cles. ${ }^{28}$ In addition, more formal reviewing methodologies for these non-traditional educational resources are emerging. Initially, there was the Approved Instructional Resources (AIR) score and the Social Medic Index (SMI), and the Medical Education Translational Resources: Impact and Quality (METRIQ) has recently been added as a quality rating tool. ${ }^{26-29}$ The AIR score is a novel scoring system that assigns a certification of quality to social media content, whereas the SMI generates a score based on website ranking and activity indicators. ${ }^{26,27}$ The most recently added METRIQ scoring system reviews eight elements of the online resource (including the content, publisher and references) and assigns a score. ${ }^{29}$

The FOAM EM site used at the time of data collection in this study endorsed the quality principles that the AIR score represents. ${ }^{26,27}$ The majority of FOAMed users are aware of this limitation and have become familiar with the top-rated FOAMed contributors. The creators and renowned FOAMed contributors remain actively involved in improving this community. Daily information is shared on how quality assurance is being improved. ${ }^{26-30}$

\section{LIMITATIONS}

The analysis of data was based on the authors' interpretation of the AEA capabilities and how these relate to the AEA refresher course outcomes. These results serve as the first step in a process towards supplementing course delivery and should ideally include a wider scope of practice and be reviewed by other professionals in the field. In addition, exact transferability of results may vary as FOAMed does not deliver the exact same topic coverage month-to-month and is instead influenced by trends in topics that have the most recent updates or changes. Some resources relevant to the AEA refresher course outcomes may have been missed due to the FOAM EM site relying on its contributors correctly tagging resources. This retrospective review only analysed FOAMed posts available in English and it is possible that, although course content is presented only in English in South Africa, having online content in other languages could be of value to South Africa's diverse population.

Recommendations for future studies include a review of FOAMed resources over a longer period of time by multiple researchers, and the application of a quality rating and scoring system. A review of specifically South African sourced FOAMed contributions may improve the context and applicability of using FOAMed resources as an educational supplement to the delivery of CPD courses. Furthermore, the methodology applied to this study could inform research methods for others in the research community, focusing on the potential connections between technology and the educational environment.

\section{CONCLUSION}

This study highlights the potential of FOAMed resources to supplement the delivery of CPD courses. In particular, the subjects of professionalism and general medical emergencies were welldescribed in the posts that were trending at the time of analysis. This suggests that FOAMed resources are not limited to clinical knowledge only, but include aspects of practitioner wellbeing. Given that outcomes are unequally represented, educational institutions wishing to make use of FOAMed to supplement the delivery of CPD courses, like the AEA refresher course, or practitioners wishing to enhance their competencies, should be aware that despite the value that FOAMed can offer, due caution must be given to the critiques and limitations of FOAMed.

\section{CONFLICTS OF INTEREST}

The authors report no conflicts of interest.

\section{AUTHOR CONTRIBUTIONS}

JS completed the study as part of a postgraduate degree and was responsible for study conceptualisation, design, analysis and interpretation of the data, drafting content and approving the version to be published. SS and LK supervised JS and contributed to all these elements. ED assisted with critical revision of content and approval of the version to be published.

\section{REFERENCES}

1. Weingart SD, Faust JS. Future evolution of traditional journals and social media medical education. Emerg Med Australas. 2014;26:626.

2. Leeuwenburg TJ, Parker C. Free open access medical education can help rural clinicians deliver 'quality care, out there. Rural Remote Health 2015;215:3185.

3. Cevik AA, Aksel G, Akoglu H, Eroglu SE, Dogan NO, Altunci YA. Social media, FOAMed in medical education and knowledge sharing: Local experiences with international perspective. Turk J Emerg Med. 2016;16:112-7. 
4. Nickson CP, Cadogan MD. Free Open Access Medical education (FOAM) for the emergency physician. Emerg Med Australas. 2014;26:76-83.

5. Diner BM, Carpenter CR, O'Connell T, et al. Graduate medical education and knowledge translation: role models, information pipelines, and practice change thresholds. Acad Emerg Med. 2007;14:1008-14.

6. Roland D, Brazil V. Top 10 ways to reconcile social media and 'traditional' education in emergency care. Emerg Med J. 2015;32:81922.

7. Hilburg R, Patel N, Ambruso S, Biewald MA, Farouk SS. Medical education during the Coronavirus Disease-2019 pandemic: Learning from a distance. Adv Chronic Kidney Dis. 2020;27:412-7.

8. Cheston CC, Flickinger TE, Chisolm MS. Social media use in medical education: A systematic review. Acad Med. 2013;88:893-901.

9. Abate LE, Gomes A, Linton A. Engaging students in active learning: use of a blog and audience response system. Med Ref Serv Q. 2011;30:12-8.

10. Dinh M, Tan T, Bein K, Hayman J, Wong YK, Dinh D. Emergency department knowledge management in the age of Web 2.0: evaluation of a new concept. Emerg Med Australas. 2011;23:46-53.

11. Stuntz R, Clontz R. An evaluation of emergency medicine core content covered by Free Open Access Medical Education resources. Ann Emerg Med. 2016;67:649-53.

12. Sinton D, Fudge J, Sillet J, Prest R, Roland D, Lewis G. G247(p) A paediatric emergency medicine (PEM) curriculum mapped Free Open Access Medical education (FOAMed) resource. Arch Dis Child. 2016;101:A134A135.

13. Silett J, Prest R, Sinton D, Roland D, Lewis G. Can we cover a curriculum with FOAM(ed) or is it full of holes? Leicester: East Midlands Emergency Medicine Education Media, 2018. (https : / / em3 . org . uk / posters-andinfographics) (visited on 09/26/2020).

14. Laal M, Salamati P. Lifelong learning; why do we need it? Procedia - Social and Behavioral Sciences 2012;31:399-403.

15. Forsetlund L, Bjorndal A, Rashidian A, et al. Continuing education meetings and workshops: effects on professional practice and health care outcomes. Cochrane Database Syst Rev. 2009:CD003030.
16. Lessing A, de Witt $M$. The value of continuous professional development: Teachers' perceptions. South African Journal of Education 2007;27:53-67.

17. Burk MJ, Bishop L, Rahman T, Koch J. Louisville Lectures: FOAMed Meets Internal Medicine Residency. Am J Med Sci. 2017;353:263-4.

18. Capabilities of emergency care providers. Pretoria: Health Professions Council of South Africa, 2018. (https : / / hpcsa . co . za / Uploads / EMB / List \%

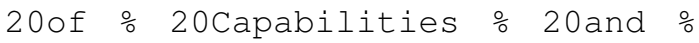
20 Medications $20 \div 20$ - 20July 202018 .pdf).

19. Krathwohl DR. A revision of Bloom's Taxonomy: An overview. Theory Into Practice 2002;41:212-8.

20. Krippendorf K. Content analysis: an introduction to its methodology. 2nd ed. Thousand Oaks: Sage Publications, 2004:xx.

21. Vaismoradi M, Turunen H, Bondas T. Content analysis and thematic analysis: Implications for conducting a qualitative descriptive study. Nurs Health Sci. 2013;15:398-405.

22. Free Open Access Medical Education curator feeds. (http : / / www . foamem . com/) (visited on $11 / 18 / 2016)$.

23. Life in the Fast Lane. the world of FOAM and EMCC. (https://litfl.com/) (visited on 06/19/2017).

24. Life in the Fast Lane. RSS feed. (https : / / feedpress . me / LITFL/) (visited on 09/26/2020).

25. Thoma B, Chan TM, Paterson QS, Milne WK, Sanders JL, Lin M. Emergency medicine and critical care blogs and podcasts: Establishing an international consensus on quality. Ann Emerg Med. 2015;66:396-402.

26. Chan TM, Grock A, Paddock M, Kulasegaram $\mathrm{K}$, Yarris LM, Lin M. Examining reliability and validity of an online score (ALiEM AIR) for rating Free Open Access Medical Education resources. Ann Emerg Med. 2016;68:72935.

27. Trueger NS, Schriger DL. Rating the ratings: The AIR scoring system for blogs and podcasts: May 2016 Annals of Emergency Medicine Journal Club. Ann Emerg Med. 2016;67:675-6. 
28. Allen NG, Chandrasekaran EB, Goett RR, Kluesner NH, Vearrier L, ACEP Ethics Committee. We must analyze and clear up the ethical issues in FOAM. Dallas: American College of Emergency Physicians, 2018. (https: / / www - acepnow . com / article / we must - analyze - and-clear - up - the ethical-issues-in-foam/) (visited on 09/26/2020).
29. Colmers-Gray IN, Krishnan K, Chan TM, et al. The revised METRIQ Score: A quality evaluation tool for online educational resources. AEM Educ Train. 2019;3:387-92.

30. Lin M, Joshi N, Grock A, et al. Approved instructional resources series: A national initiative to identify quality emergency medicine blog and podcast content for resident education. J Grad Med Educ. 2016;8:219-25. 